\title{
ABC transporters and NR4A1 identify a quiescent subset of tissue-resident memory T cells
}

\author{
Chandra Sekhar Boddupalli, ${ }^{1}$ Shiny Nair, ${ }^{1}$ Simon M. Gray, ${ }^{2}$ Heba N. Nowyhed, ${ }^{3}$ Rakesh Verma, ${ }^{1}$ Joanna A. Gibson, ${ }^{4}$ \\ Clara Abraham, ${ }^{1}$ Deepak Narayan, ${ }^{5}$ Juan Vasquez, ${ }^{6}$ Catherine C. Hedrick, ${ }^{3}$ Richard A. Flavell, ${ }^{2,7}$ Kavita M. Dhodapkar, ${ }^{6}$ \\ Susan M. Kaech, ${ }^{2,7}$ and Madhav V. Dhodapkar ${ }^{1,2}$ \\ 'Department of Medicine and 2Department of Immunobiology, Yale School of Medicine, New Haven, Connecticut, USA. ${ }^{3}$ La Jolla Institute for Allergy and Immunology, Division of Inflammation Biology, \\ La Jolla, California, USA. ${ }^{4}$ Department of Pathology, ${ }^{5}$ Department of Surgery, ${ }^{6}$ Department of Pediatrics, and ${ }^{7}$ Howard Hughes Medical Institute, Yale School of Medicine, New Haven, Connecticut, USA.
}

\begin{abstract}
Immune surveillance in tissues is mediated by a long-lived subset of tissue-resident memory T cells (Trm cells). A putative subset of tissue-resident long-lived stem cells is characterized by the ability to efflux Hoechst dyes and is referred to as side population (SP) cells. Here, we have characterized a subset of SP T cells (Tsp cells) that exhibit a quiescent ( $G_{0}$ ) phenotype in humans and mice. Human Trm cells in the gut and BM were enriched in Tsp cells that were predominantly in the $G_{0}$ stage of the cell cycle. Moreover, in histone 2B-GFP mice, the 2B-GFP label was retained in Tsp cells, indicative of a slow-cycling phenotype. Human Tsp cells displayed a distinct gene-expression profile that was enriched for genes overexpressed in Trm cells. In mice, proteins encoded by Tsp signature genes, including nuclear receptor subfamily 4 group A member 1 (NR4A1) and ATP-binding cassette (ABC) transporters, influenced the function and differentiation of Trm cells. Responses to adoptive transfer of human Tsp cells into immune-deficient mice and plerixafor therapy suggested that human Tsp cell mobilization could be manipulated as a potential cellular therapy. These data identify a distinct subset of human T cells with a quiescent/ slow-cycling phenotype, propensity for tissue enrichment, and potential to mobilize into circulation, which may be harnessed for adoptive cellular therapy.
\end{abstract}

\section{Introduction}

A fundamental property of human $\mathrm{T}$ cells is to provide lifelong immunity against pathogens lasting several decades (1). The complement of $\mathrm{T}$ cells within an individual is heterogeneous and includes naive $\mathrm{T}$ cells that develop from thymic precursors, shortlived effector cells, and memory T cells derived from naive T cells following antigen exposure. Maintenance of each of these components for several decades of life is essential to host defense: memory $\mathrm{T}$ cells for defense against previously encountered antigens and naive $\mathrm{T}$ cells for responses to new antigens. T cell memory is mediated by coordinated action of distinct subsets. Initial pioneering studies classified human memory $\mathrm{T}$ cells into effector memory T cells (Tem cells) and central memory T cells (Tcm cells) based on rapid effector function and the expression of lymphoid homing receptors, respectively (2). More recent studies have identified and characterized a subset of memory $\mathrm{T}$ cells resident in nonlymphoid tissues (NLT) that mediate regional immune surveillance against pathogens (3-6). Tissue-resident memory T cells (Trm cells) in NLTs outnumber memory CD8 ${ }^{+} \mathrm{T}$ cells in lymphoid tissues and represent the primary steady-state surveillance mechanism in NLTs (7). Both Tcm and Trm cells originate from a common clonal precursor, but following tissue localization, Trm cells maintain a highly regional network and persist over decades of life with apparently little homeostatic turnover (8-10). Little is known

Conflict of interest: The authors have declared that no conflict of interest exists. Submitted: November 2, 2015; Accepted: August 4, 2016.

Reference information: J Clin Invest. 2016;126(10):3905-3916. doi:10.1172/JCI85329. about the mechanisms that underlie the development, dormancy, and maintenance of these long-lived Trm cells, particularly in humans, and investigation of these areas will have major implications for understanding immune homeostasis within tissues.

Homeostasis in adult tissues is maintained by the differentiation of a small population of adult stem cells. The capacity of $\mathrm{T}$ cells for long-term survival, quiescence, clonal differentiation, and self-renewal has elicited comparisons between long-lived postmitotic cells, such as memory T cells and adult stem cells (11). Indeed, prior studies have shown that murine memory T cells share a transcriptional program of self-renewal with long-term hematopoietic stem cells (HSCs) (12). Goodell et al. described the ability to efflux lipophilic Hoechst dyes as a distinct property of a subset of HSCs, termed side population (SP) cells, with enhanced regenerative potential $(13,14)$. The SP phenotype (i.e., dye efflux) is mediated by the expression of ATP-binding cassette (ABC) transporters (particularly ABCG2 in most HSCs and some adult stem cells), and cells with SP phenotype have been identified in putative stem cells in diverse tissues (15-18). ABC transporters efflux diverse substrates, and their expression in stem cells is postulated to protect these cells from xenotoxic, oxidative, and metabolic stress $(15,19)$. Human CD $8^{+}$memory T cells expressing P-glycoprotein/ $\mathrm{ABCB} 1$ were shown to persist following cancer chemotherapy (20); however, most human $\mathrm{T}$ cells with $\mathrm{ABCB}^{+}$phenotype were later shown to be mucosa-associated invariant T (MAIT) cells (21). We hypothesized that human T cells with SP phenotype may identify a subset with distinct biologic and functional properties. Here, we show that SP phenotype marks a distinct subset of human and 
A

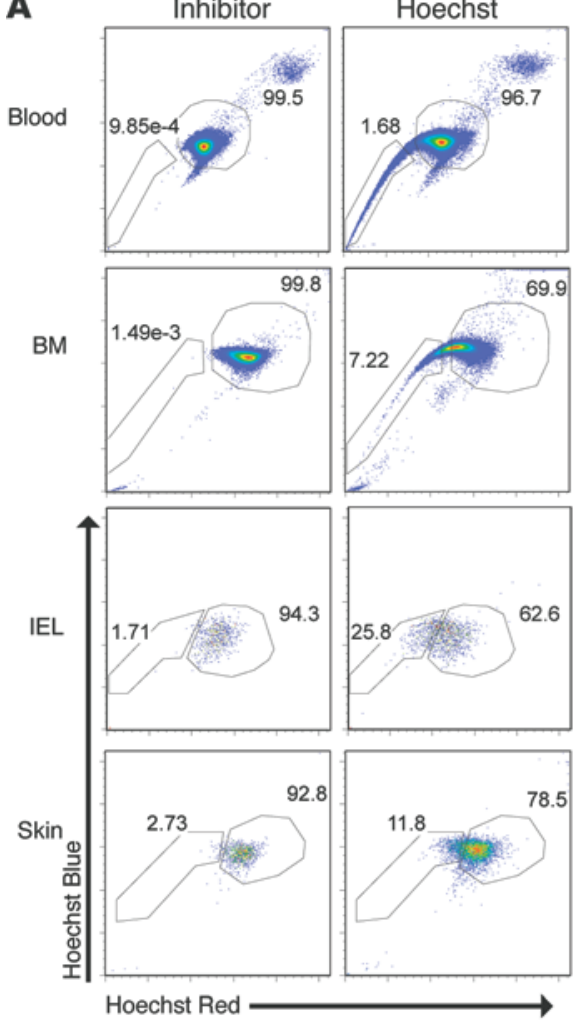

B

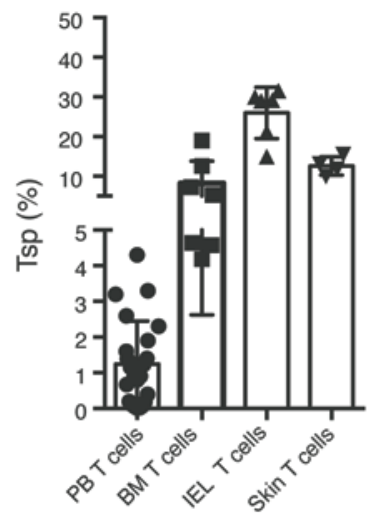

C

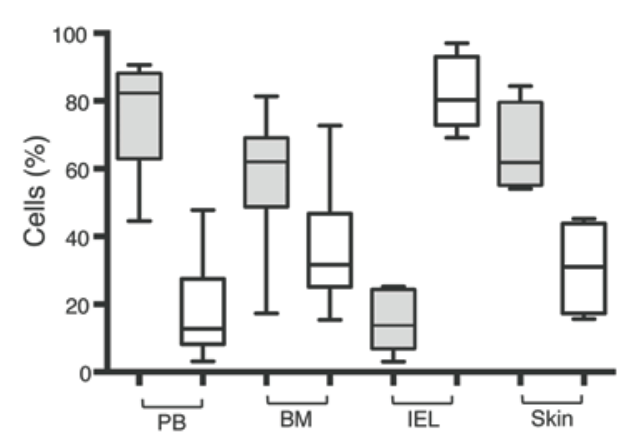

$\square$ CD4 Tsp cells

$\square$ CD8 Tsp cells

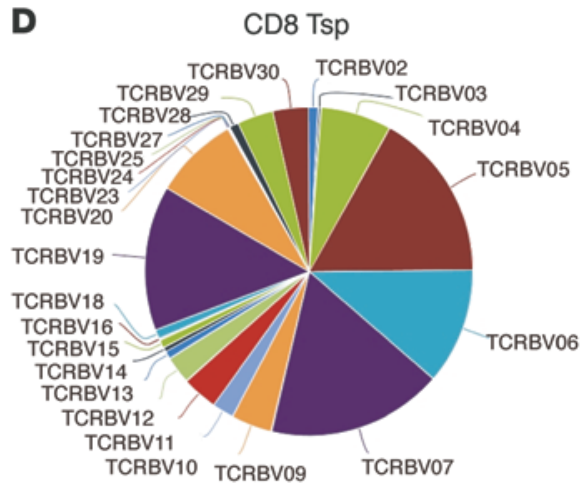

E

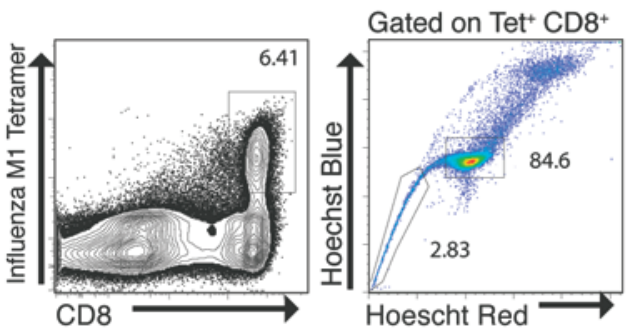

$\mathbf{F}$
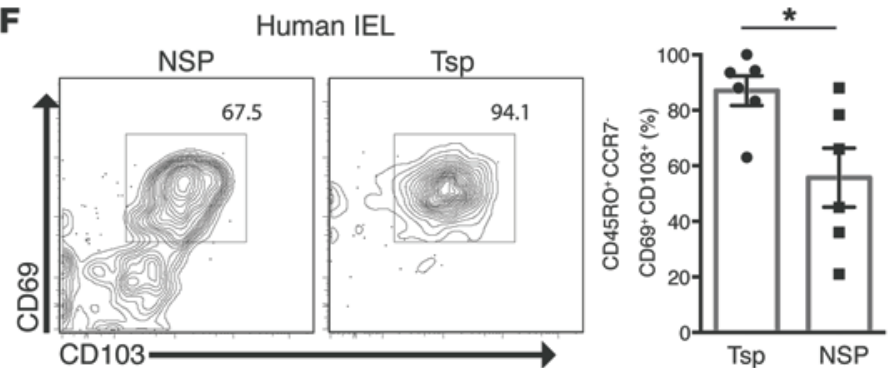

G

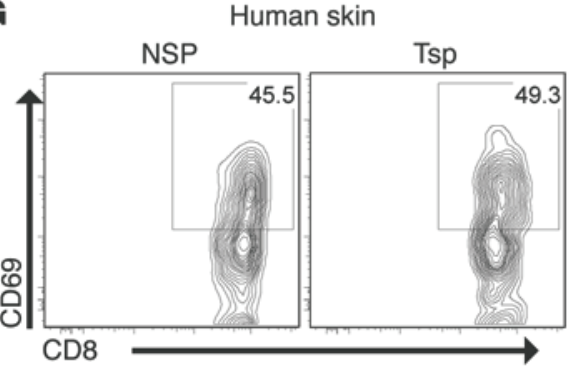

H

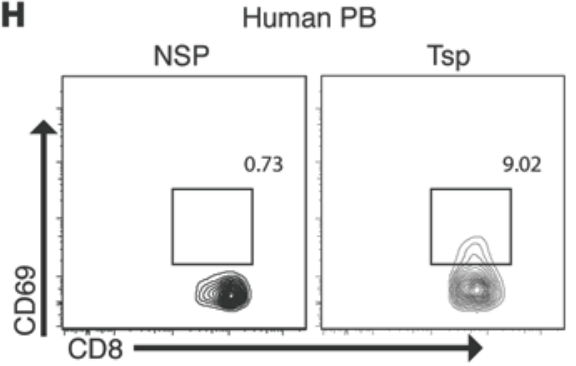

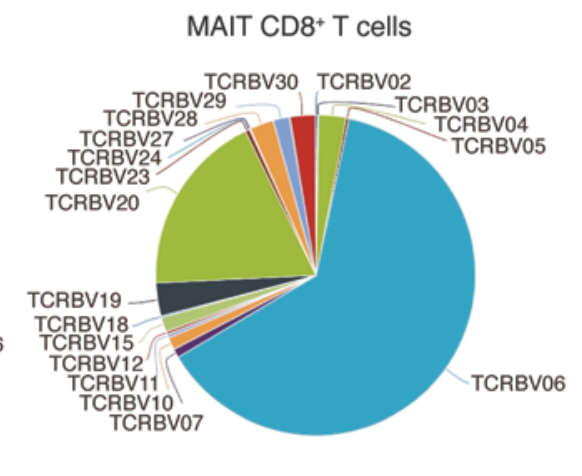

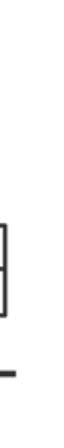


murine T cells. Trm cells in human and murine tissues such as the gut are highly enriched SP T cells (Tsp cells), and these cells particularly mark a Trm subset with quiescent/slow-cycling phenotype. Human Tsp cells share overlapping transcriptional gene-expression programs with Trm cells (10), including several members of the NR4A orphan nuclear receptor family, also implicated in HSC quiescence $(22,23)$. We also show that 2 key signature genes identified in human Tsp cells, ABC transporters and nuclear receptor subfamily 4 group A member 1 (NR4A1), affect the biology of Trm cells. Harnessing the properties of Tsp cells may therefore allow manipulation of tissue-resident memory in humans. Their quiescence, propensity for tissue localization, and potential to mobilize into circulation also make them attractive candidates for adoptive cellular therapies targeting cancer.

\section{Results}

Detection of human Tsp cells. In order to detect human Tsp cells, freshly isolated human T cells from blood, BM, gut, and skin were analyzed for the ability to efflux Hoechst dye. Prior treatment with verapamil prevented dye efflux and was utilized as a control. Tsp cells could be identified in circulating T cells, but were detected at a much higher frequency in the BM, intestinal epithelium, and skin (Figure 1, A and B). The proportion of $\mathrm{CD} 4^{+} \mathrm{CD} 8^{+}$Tsp cells with naive/memory phenotype in blood, BM, skin, and intestinal epithelial lymphocytes (IEL) was similar to that in the nonside population (NSP) cells (Figure 1C and Supplemental Figure 1, A-D; supplemental material available online with this article; doi:10.1172/JCI85329DS1). T cell receptor (TCR) sequencing of purified Tsp cells documented the presence of diverse repertoires as opposed to MAIT cells with restricted TCRs (Figure 1D). The presence of Tsp phenotype was also detected within the human influenza-matrix peptide-specific memory $\mathrm{T}$ cells (Figure 1E). Nearly all Tsp cells in the gut were $\mathrm{CD} 9^{+} \mathrm{CD} 103^{+}$, consistent with Trm $\mathrm{T}$ cell phenotypes (Figure $1 \mathrm{~F}$ ). Similar enrichment of CD69 ${ }^{+}$CCR7- ${ }^{-}$Trm cells was not observed in skin CD8 ${ }^{+}$Tsp cells (Figure 1G). In contrast to $\mathrm{T}$ cells within tissues, only a small fraction of $\mathrm{T}$ cells within human peripheral blood mononuclear cells (PBMCs) express CD69. Interestingly, these circulating CD69+ human $\mathrm{T}$ cells are enriched within Tsp cells and have a phenotype of CCR7- CD62L- CD45RO+ CD45RA- IL7R ${ }^{\text {lo }} \mathrm{T}$ cells (Figure $1 \mathrm{H}$ ). Taken together, these results show that human Tsp cells have a diverse phenotype and can be detected in several tissues, but are particularly enriched in Trm cells in tissues such as the gut.

Enrichment of Trm phenotype within murine Tsp cells. We next evaluated the generation and tissue localization of murine CD8 ${ }^{+}$ memory Tsp cells using a lymphocytic choriomeningitis virus (LCMV) infection model. Thy $1.1^{+} \mathrm{P}^{+} 4^{+} \mathrm{CD}^{+} \mathrm{T}$ cells were transferred into naive C57BL/6 (B6) mice and analyzed at different time points following LCMV infection. Tsp CD $8^{+} \mathrm{T}$ cells were initially detected in the lymphoid tissues at day 8 following infection, but declined by day 30 (Supplemental Figure 2A). In contrast, enrichment of Tsp cells was detected in the BM up to 60 days following infection (Supplemental Figure 2A). Detailed analysis of tissues at 30 days following infection revealed that Tsp cells were enriched within the $\mathrm{BM}$, liver, and the gut, but not the spleen (Supplemental Figure 2B). Interestingly, $\mathrm{P} 14^{+} \mathrm{CD} 8^{+}$Tsp cells were mostly confined to $\mathrm{CD} 69^{+} \mathrm{T}$ cells in the liver and $\mathrm{CD} 69^{+} \mathrm{CD} 103^{+} \mathrm{T}$ cells in Peyer's patches (PP), IEL, and lamina propria lymphocytes (LPL) (Supplemental Figure 2C). Enrichment of Tsp cells was also documented within gp33-tetramer ${ }^{+} \mathrm{T}$ cells in the gut (Figure 2, A and B), and the majority of gut-resident Tsp cells were enriched for Trm phenotype $\left(\mathrm{CD} 69^{+} \mathrm{CD} 103^{+}\right)$(Figure 2, C and D). In accordance with this finding, we also observed similar enrichment of $\mathrm{CD}^{+}$Tsp cells in human gut Trm cells (Supplemental Figure 2D). Taken together, these data show that antigen-specific murine Tsp memory $\mathrm{CD}^{+} \mathrm{T}$ cells emerge at an early stage of immune response and progressively accumulate in the mucosal tissues enriched for Trm phenotype.

Slow-cycling phenotype of human and murine Tsp cells. In order to ascertain whether Tsp cells represent a functionally distinct subset of human/murine T cells, we compared their cell-cycle status with NSP counterparts. SP phenotype cells in HSCs and adult tissue stem cells are characterized as being in a noncycling state $\left(G_{0}\right.$ phase) of the cell cycle (24). Nearly all of the $C D 4^{+}$and $\mathrm{CD}^{+}$Tsp cells in human PBMCs were enriched in the $G_{0}$ phase of the cell cycle compared with NSP cells based on Hoechst and Pyronin Y staining (Figure 3, A and B). As with circulating human Tsp cells, those in the BM were also predominantly in the $G_{0}$ stage of the cell cycle (data not shown). Importantly, the CD8 ${ }^{+}$Tsp subsets of Trm cells from human gut and skin were also enriched in the $G_{0}$ phase of the cell cycle compared with $C D 8^{+}$NSP Trm cells within these tissues (Figure 3, A and B). This low-cycling phenotype of Tsp cells was also observed within murine Trm cells. At 35 days following LCMV infection, nearly all GP33-tetramer ${ }^{+} \mathrm{CD}^{+}$ Trm cells with Tsp phenotype in PP, IEL, and LPL were in $G_{0}$, while NSP counterparts were in both the $G_{0}$ and $G_{1}$ phases of the cell cycle (Figure 3C).

Pyronin Y staining represents only a snapshot of cycling status. Label retention in doxycycline-inducible (Dox-inducible) histone H2B GFP mice has been utilized to identify quiescent/ slow-cycling populations of stem cells in adult tissues $(25,26)$, but to our knowledge, not yet comparably applied for the evaluation of the quiescent compartment of murine memory $\mathrm{T}$ cells in vivo. Mice fed with Dox for 2 weeks were infected with LCMV in the presence of Dox for up to 6 weeks after infection, and subsequently, a 5-week chase was performed by removal of Dox treatment (Figure 3D). Uniform labeling of $\mathrm{CD} 8^{+} \mathrm{T}$ cells expressing GFP prior to chase was verified by flow cytometry (Supplemental Figure 3A). This strategy revealed that the majority of the lymphoid memory $\mathrm{CD}^{+} \mathrm{T}$ cells in the spleen, liver, or BM have undergone cycling and are $\mathrm{GFP}^{-}$with very few GFP label-retaining $\mathrm{CD}^{+} \mathrm{T}$ cells (Figure 3E). In contrast, label-retaining $\mathrm{CD}^{+}$ memory T cells could be detected in the PP and IEL (Figure 3E). Also, as expected, a higher percentage of GFP label-retaining cells were found in the $\mathrm{c}-\mathrm{Kit}^{+}$stem and progenitor cells in the BM as well as in naive CD8 ${ }^{+} \mathrm{T}$ cells in the spleen (Supplemental Figure $3 \mathrm{~B})$. As Tsp cells were particularly enriched within the CD8 ${ }^{+}$ gut Trm cells, we analyzed the proportion of label-retaining cells at the end of a 5-week chase within the Tsp or NSP compartments of PP or IEL Trm cells. SP CD ${ }^{+}$Trm cells from PP and IEL had a significantly higher proportion of label-retaining cells compared with NSP cells (Figure 3, F and G). In contrast with murine tissues and human blood, we were unable to detect Tsp cells in murine blood (Supplemental Figure 3C). Together, these data identify 
A

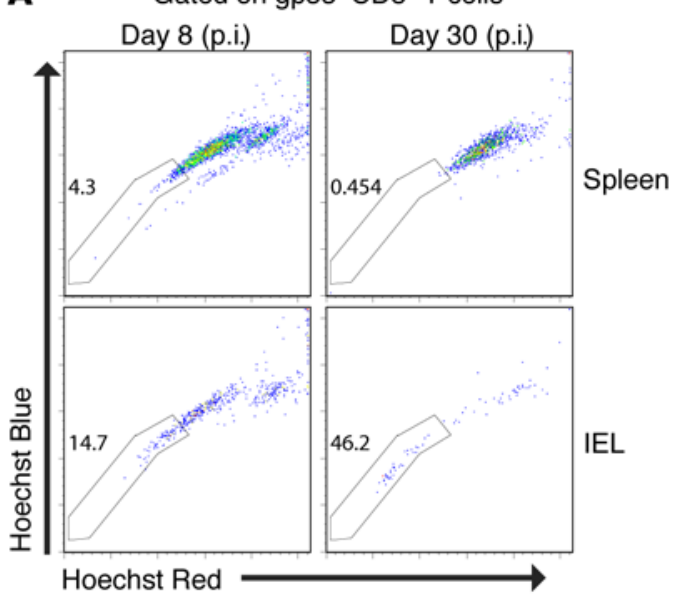

B

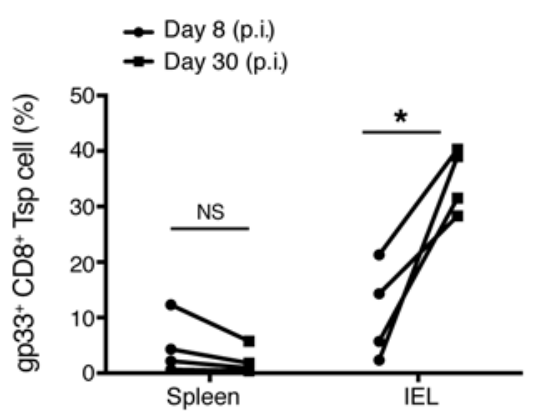

Figure 2. SP phenotype marks LCMV-specific CD8 ${ }^{+}$Trm cells. (A) Representative CD8 ${ }^{+}$Tsp analysis on LCMVArm-infected mouse spleen and IEL at day 8 (left) and day 30 (right). p.i., post infection. (B) Graph representing the analysis in $\mathbf{A}$ ( $n=4$ mice). Experiment was repeated twice; graph here represents one of the experiments. (C) Top panel shows percentage of CD8 ${ }^{+}$ Tsp and NSP gp33+CD8 ${ }^{+} T$ cells at day 35 after infection in different organs. Bottom panel is gated on gp $33^{+}{ }^{+} D 8^{+}$ Tsp cells. (D) Percentage of gp $33^{+}$Trm cells in CD8 ${ }^{+}$Tsp and NSP fractions. Bar graphs represent data from 7-10 mice. Data were compiled from 3 independent experiments. ${ }^{*} P<0.05$, ${ }^{* *} P<$ $0.01,{ }^{* *} P<0.001$, by Student's $t$ test.
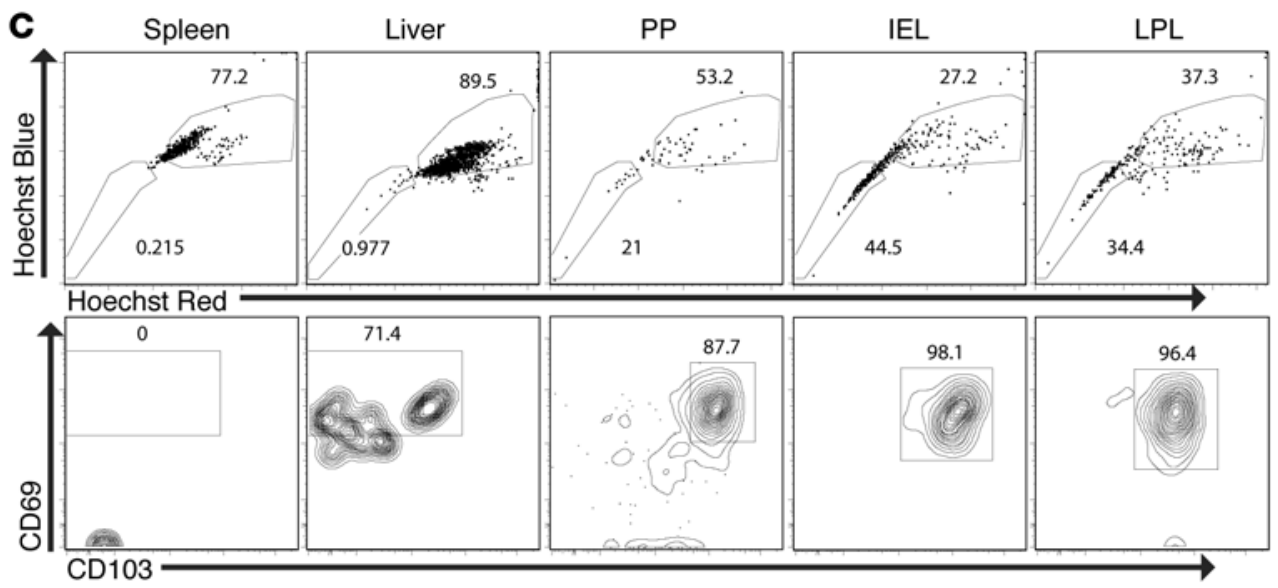

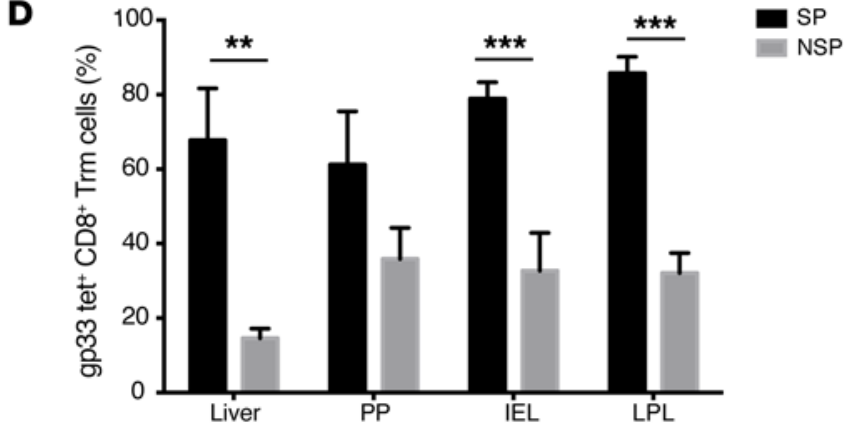

a distinct slow-cycling compartment within gut Trm cells and demonstrate that the Tsp phenotype identifies a functionally distinct subset of human/murine T cells with a slow-cycling phenotype within the Trm compartment.

Distinct gene-expression profile in human $C D 8^{+}$Tsp cells. In order to better understand the functional properties of Tsp cells, we compared the transcriptome of purified human CD8 ${ }^{+}$Tsp cells to that of $\mathrm{CD}^{+}$naive, Tcm, and Tem cells isolated from the same donor. Principal component analysis (PCA) revealed that Tsp cells had a distinct gene-expression profile that was more closely related to that of Tem $\mathrm{CD}^{+} \mathrm{T}$ cells than $\mathrm{Tcm}$ or naive $\mathrm{CD}^{+} \mathrm{T}$ cells (Figure 4A and Supplemental Figure 4). Discriminant analysis for genes differentially regulated within the $\mathrm{CD} 8^{+}$Tsp compartment as compared with other $\mathrm{CD}^{+} \mathrm{T}$ cell subsets identified 119 tran- scripts that formed a core CD $8^{+}$Tsp signature (Figure 4B, Supplemental Figure 4 and Supplemental Table 1). MetaCore pathway analysis for $G_{0}$ terms revealed that the top Tsp signature-related pathways were metabolic regulation and cell cycle (Figure 4C). Similar analysis for $\mathrm{CD} 4^{+}$Tsp cells revealed that the genes overexpressed within $\mathrm{CD} 4^{+}$Tsp cells were highly overlapping with those in $\mathrm{CD}^{+}$Tsp cells (data not shown). Several of the genes overexpressed in Tsp cells are associated with Trm cells, and genes comprising the recently described core signature of human skin Trm cells (27) were increased in $\mathrm{CD}^{+} \mathrm{Tsp}$ cells compared with naive, $\mathrm{CD}^{+} \mathrm{Tem}$, and Tcm counterparts (Figure 4D). This core human $\mathrm{CD}^{+}$Tsp signature included XCL1, SKIL, SIK1, and several members of nuclear hormone family of transcription factors $N R 4 A 1$ (also known as NUR77), NR4A2, and NR4A3, which are also over- 
A

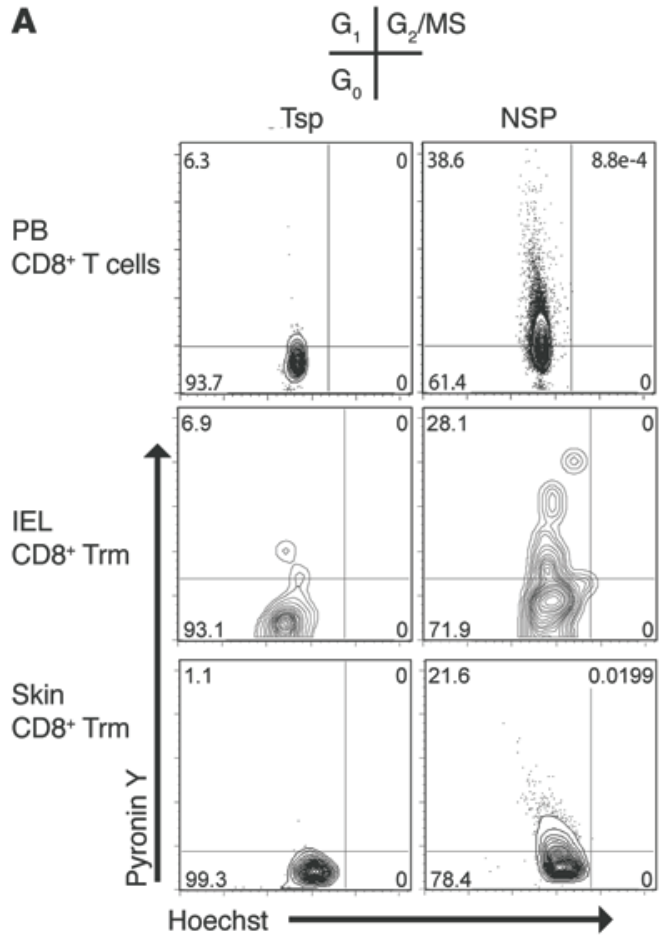

B

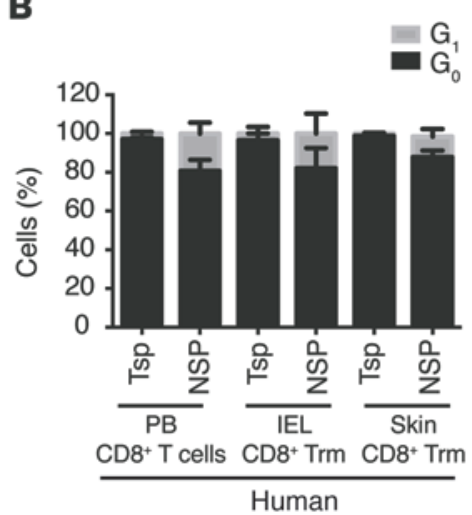

C

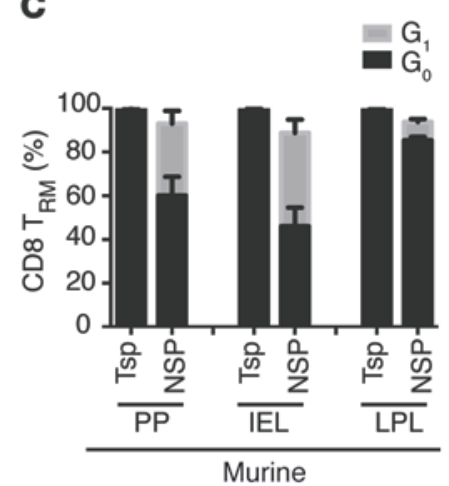

D H2B-GFP mice

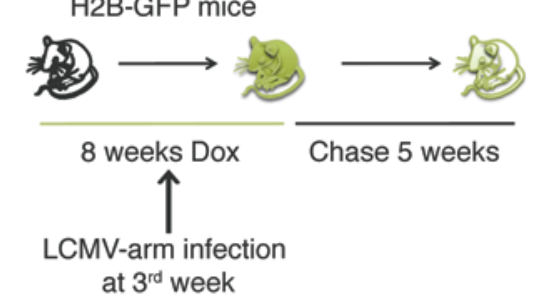

E
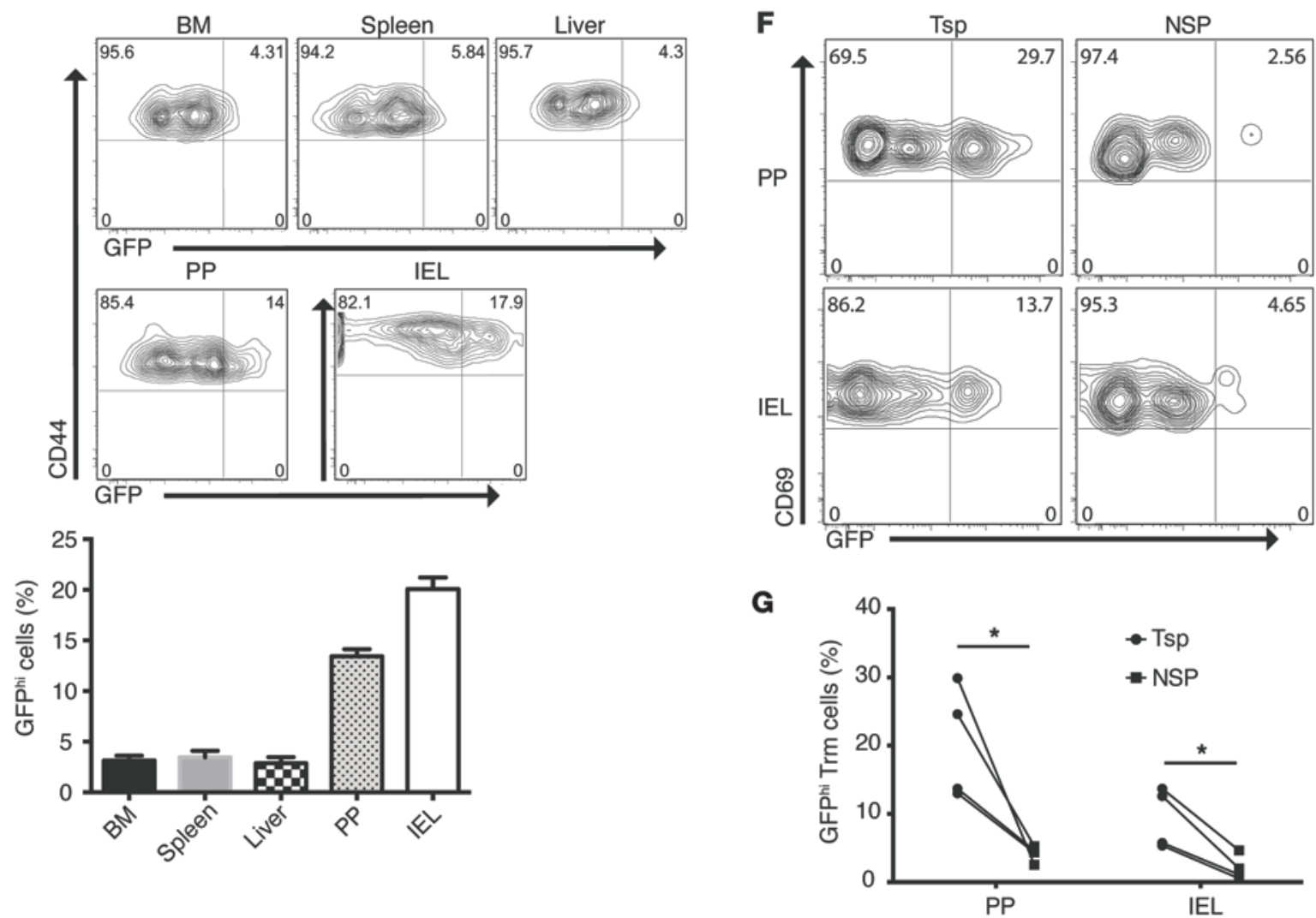

Figure 3. Cell-cycle analysis of Tsp cells. (A) Hoechst-Pyronin Y cell-cycle analysis on Tsp and NSP fraction from human blood CD8 ${ }^{+} \mathrm{T}$ cells (top panel), IEL (middle panel), and skin CD8 ${ }^{+}$Trm cells (bottom panel). Result is representative of 3 independent experiments. (B) Bar graph represents combined data of 3 independent experiments. (C) Bar graph displays percentage of $G_{0}$ and $G_{1}$ fractions in CD8 ${ }^{+}$Tsp and NSP gated on Trm compartment from PP, IEL in mice at day 35 (p.i.) with LCMV-Arm. (D) Schematic diagram showing the protocol for measuring GFP label retention in H2B-GFP mice upon LCMV-Arm infection. (E) FACS analysis on memory CD8 ${ }^{+} \mathrm{T}$ cells from BM, spleen, liver, IEL, and PP after 5 weeks of chase; the same is documented in the bar graph ( $n=4-5$ mice). (F) FACS analysis of GFP label retention in SP and NSP CD8 ${ }^{+}$Trm compartment at 5 weeks chase in H2B GFP mice infected with LCMVArm. (G) Graph represents percentages of GFP+ cells in SP and NSP CD8 ${ }^{+}$Trm cells. ${ }^{*} P<0.05$. Mouse experiments were repeated twice; graphs represent analysis from 1 experiment ( $n=4$ mice). 
A

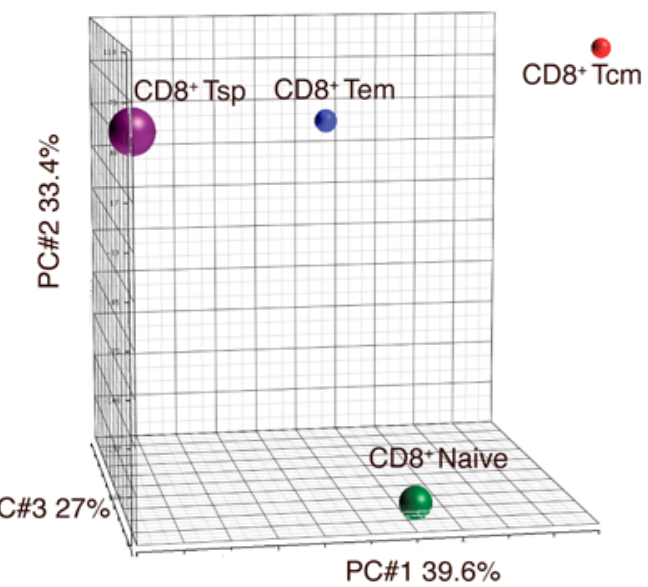

B $\quad C D 8^{+}$Tsp vs. naive $\mathrm{CD}^{+} \quad \mathrm{CD} 8^{+} \mathrm{Tsp}$ vs. EM CD8 ${ }^{+}$ 599 entities 277 entities

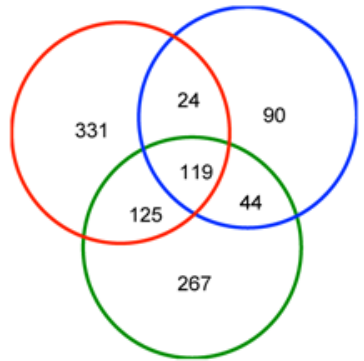

CD8+ Tsp vs. CM CD8+ 555 entities
C

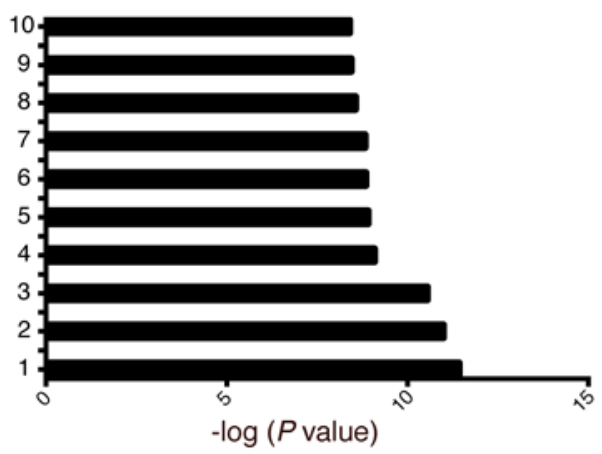

1. Primary metabolic process

2. Cellular metabolic process

3. Macromolecule metabolic process

4. Metabolic process

5. Apoptotic process

6. Nucleobase-containing compound metabolic process

7. Programmed cell death

8. Cell Cycle

9. Nitrogen compound metabolic process

10. Positive regulation of biological process
D

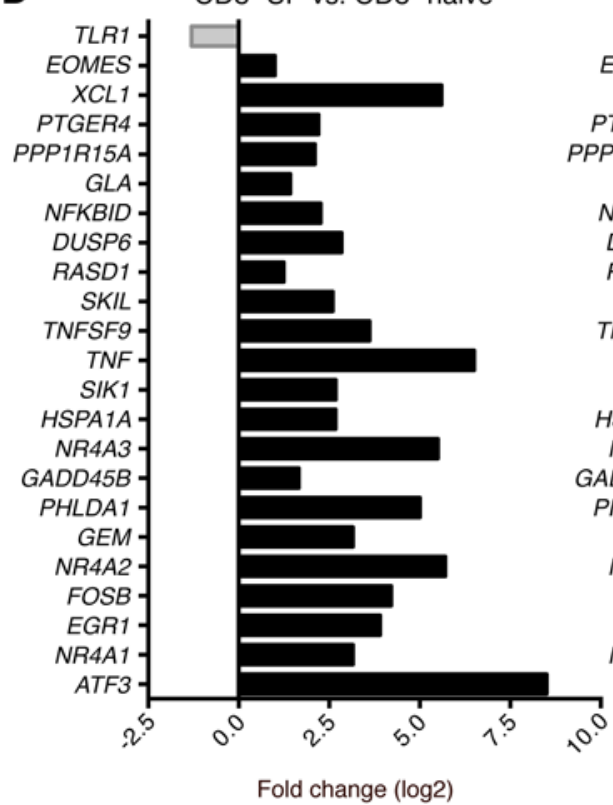

$\mathrm{CD} 8^{+} \mathrm{SP}$ vs. CD8+ EM

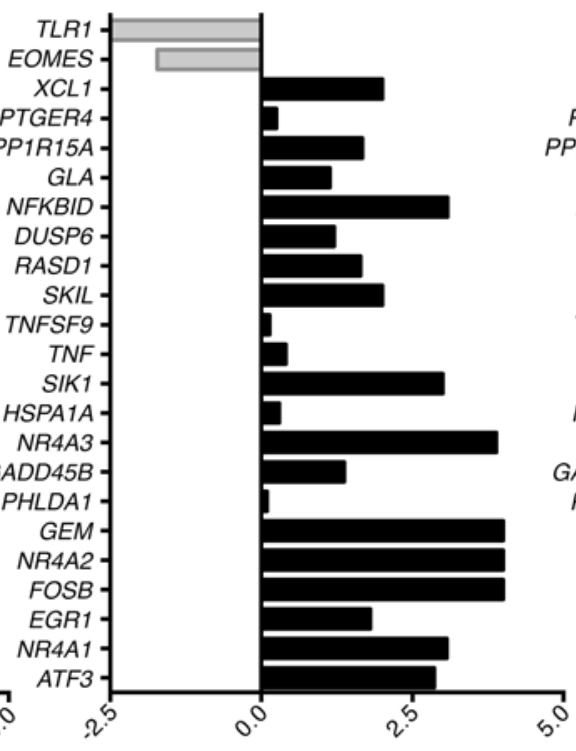

Fold change (log2)
$\mathrm{CD}^{+} \mathrm{SP}$ vs. $\mathrm{CD} 8^{+} \mathrm{CM}$

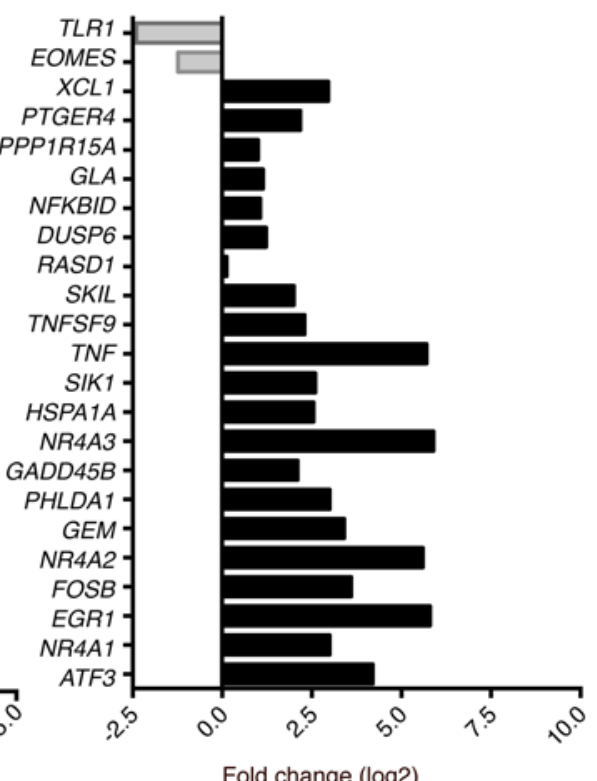

Figure 4. Human CD8+ Tsp cells express a distinct gene-expression profile. (A) PCA plot comparing CD8 ${ }^{+}$naive, $C D 8^{+} \mathrm{Tem}^{\mathrm{C}} \mathrm{CD} 8^{+} \mathrm{Tcm}$, and $\mathrm{CD} 8^{+} \mathrm{Tsp}$ cells. (B) Venn diagram of differentially regulated transcripts (>4-fold) in CD8 ${ }^{+}$Tsp compared with naive, Tem, and Tcm CD8 ${ }^{+} T_{\text {cells. }}$ (C) Top $10 \mathrm{C}_{0}$ terms from MetaCore pathway analysis of genes enriched in CD8 ${ }^{+}$Tsp cells. (D) Relative expression of human skin Trm genes in blood CD8 $8^{+}$Tsp cells compared with naive, Tem, and Tcm CD8 ${ }^{+}$T cells. 
A
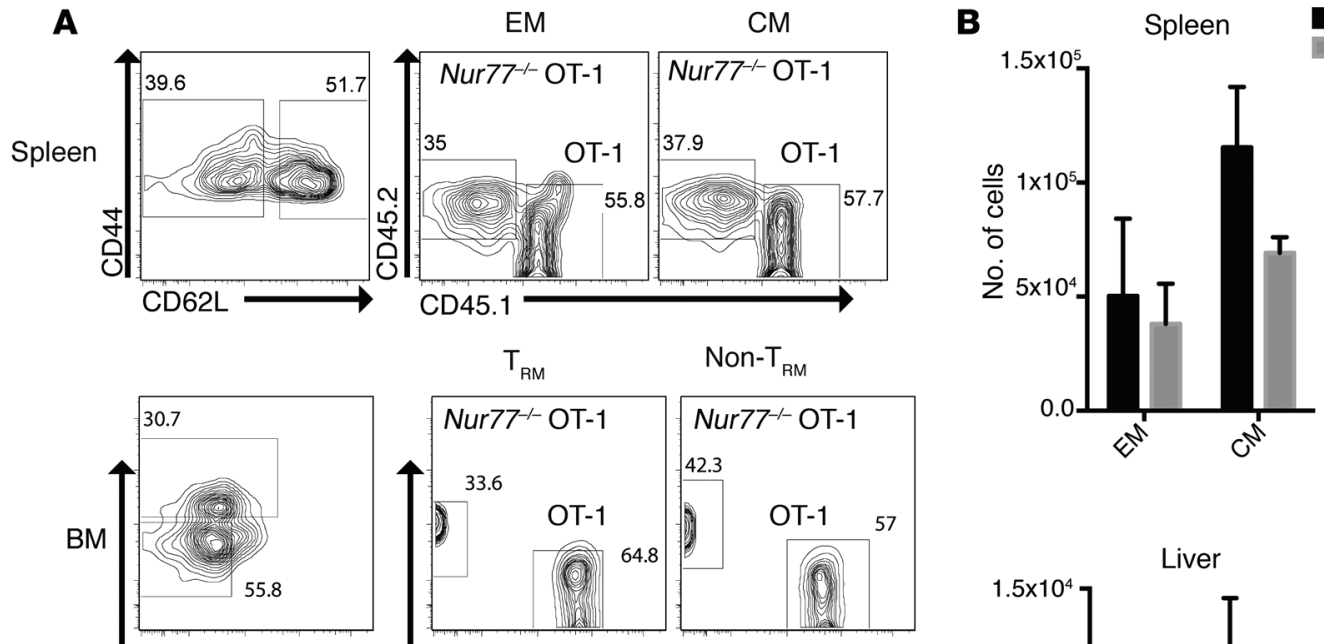

Liver
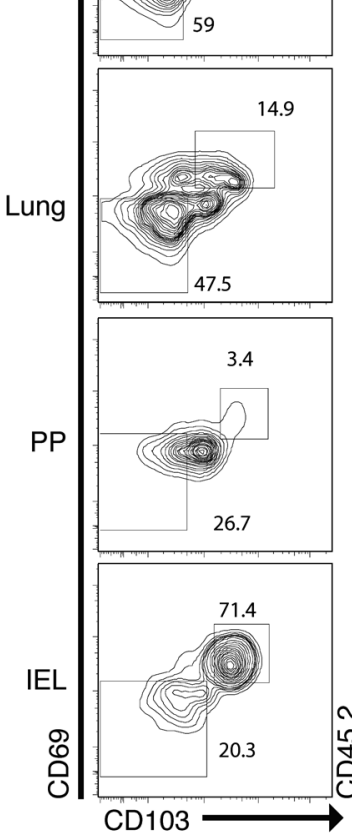

B
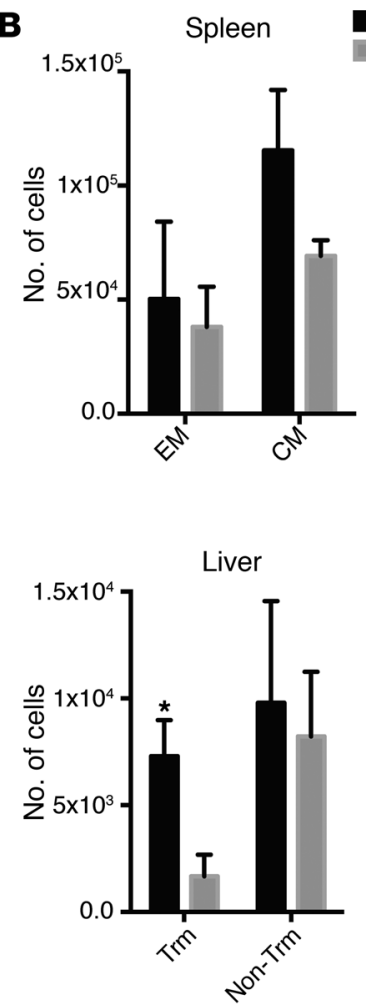

PP

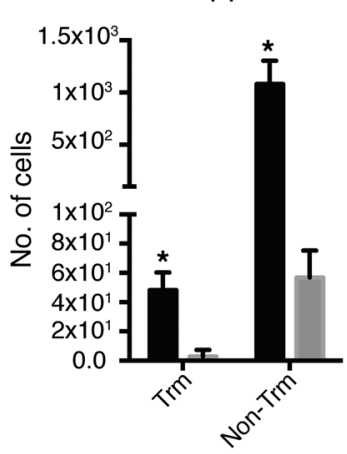

OT-1 Nur77 $7^{--}$OT-1

BM
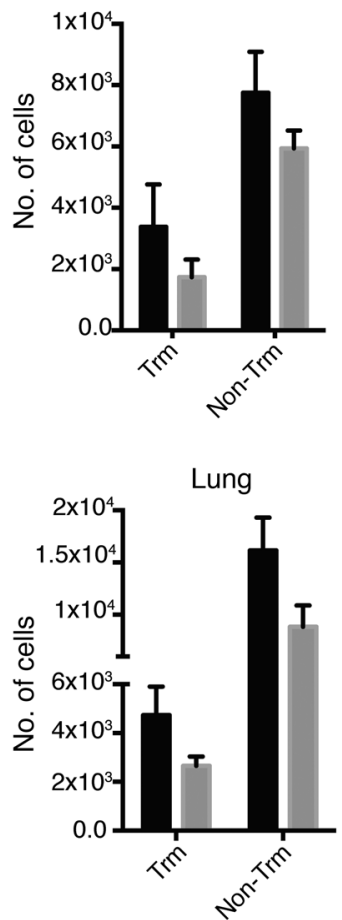

IEL

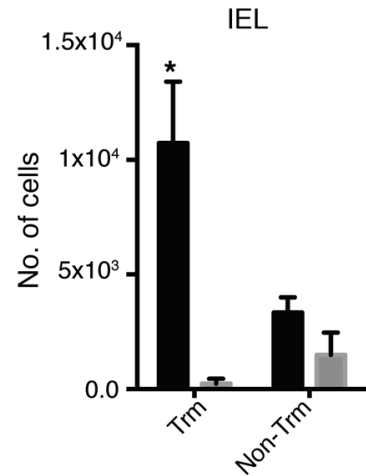

Figure 5. Impact of Nur77 on CD8 ${ }^{+}$Trm cells. Flow cytometry on $V \alpha 2 C D 8^{+} T$ cells obtained from chimeras generated by reconstitution of WT mice with 1:1 mixture of WT OT-1 and Nur77-/- OT-1 cells and then assessed at day 35 after infection with influenza X-31 OVA. (A) FACS analysis showing CD45.2 (Nur77-1OT-1) and CD45.1 (WT OT-1) cells in spleen, BM, liver, lung, PP, and IEL. (B) Bar graphs represent absolute number of Nur77-1- OT-1 and WT OT-1 CD8 ${ }^{+}$T cells from different organs. Experiments were repeated twice with 3 to 4 mice in each group; graphs represent data from 1 independent experiment ( $n=4$ mice). ${ }^{*} P<0.05$, by Student's $t$ test.

lapping with the genomic signature of murine $\mathrm{CD} 8^{+} \mathrm{Trm}$ cells (10) (Figure 4D). Shared enrichment of NR4A and ABC transporters in Tsp and Trm cells led us to test the hypothesis that these genes may play an important role in the biology of Trm cells.

Effect of NR4A1 on CD8 Trm cells. The NR4A family of transcription factors is implicated in the regulation of $\mathrm{T}$ cell development as well as quiescence of HSCs, including the subset of HSCs with SP phenotype $(22,23,28,29)$. As these genes also appear to be specifically overexpressed in Tsp and Trm cells, we analyzed the role of NR4A1 in the generation of Trm cells using adoptive transfer of NR4A1-deficient $\mathrm{T}$ cells (herein referred to as Nur77--/) in an influenza infection model. $\mathrm{CD} 8^{+} \mathrm{T}$ cell chimeras were generated by transferring V $\alpha 2$-naive $\mathrm{CD} 8^{+}$cells from CD 45.1 WT-OT-1 and CD45.2 Nur77-- OT-1 cells at a 1:1 ratio, and these mice were infected with OVA-expressing influenza virus. Both WT and Nur77-- OT-1 CD $8^{+} \mathrm{T}$ cells expanded comparably in the early (days 8-14) effector phase (Supplemental Figure 5, A and B), but the proportion of Nur77-- $\mathrm{CD} 8^{+} \mathrm{T}$ cells was reduced by day 35 following infection. The differences between Nur $77^{--}$and WT chimerism were most profound in the Trm compartment of liver, PP, and IEL, 

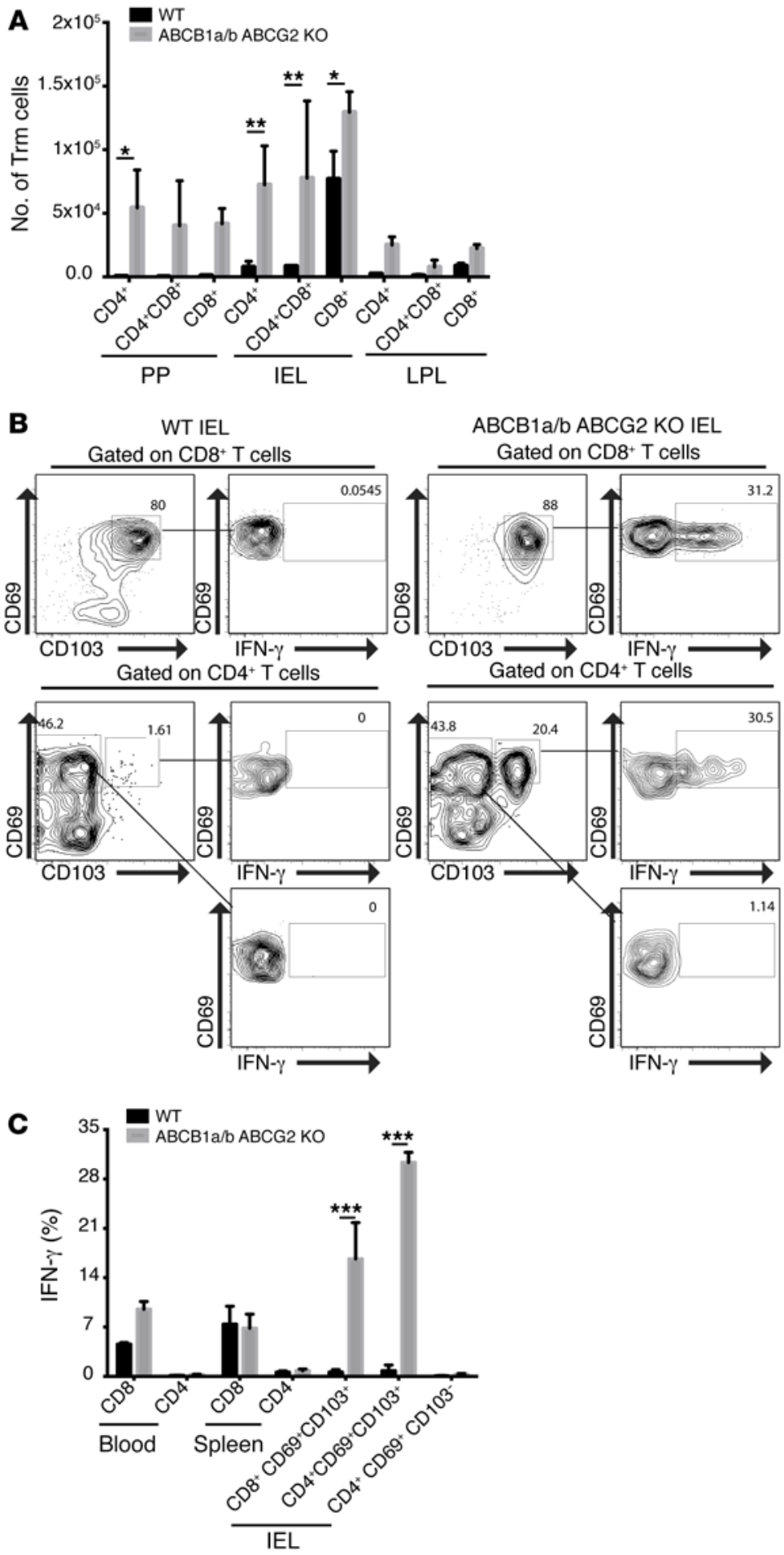

with minimal effects on non-Trm cells except in the PP (Figure 5, A and B). Together, these data suggest that NR4A1 plays an important role in regulating tissue residence of murine $\mathrm{CD} 8^{+}$Trm cells.

Alterations in gut Trm cells in ABC transporter-deficient mice. As the Tsp phenotype primarily depends on the expression of $A B C$

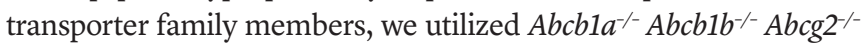
mice (herein referred to as ABCB1a/b ABCG2 KO mice) to evaluate the role of these transporters in Trm biology. As Hoechst dye exclusion depends on these transporters, Tsp phenotype cannot be detect-
Figure 6. ABCB1a/b ABCG2 KO mice display increased inflammatory Trm compartment. (A) Bar graph comparing the CD4+, $\mathrm{CD}^{+} \mathrm{CD8}^{+}$, and $\mathrm{CD} 8^{+}$Trm compartments in the gut (PP, IEL, and $\mathrm{LPL}$ ) of WT and ABCB1a/b ABCC2 KO mice. (B) Intracellular cytokine flow cytometry documenting IFN- $\gamma$ production upon PMA plus ionomycin activation of WT and ABCB1a/b ABCG2 KO mouse IELs. (C) Bar graph represents IFN- $\gamma$ production in different T cell populations from WT and ABCB1a/b ABCG2 KO mice. Experiments were repeated twice with 3 to 4 mice in each group; graphs represent data from 1 experiment $\left(n=4\right.$ mice). ${ }^{*} P<0.05$, ${ }^{* *} P<0.01,{ }^{* *} P<0.001$, by Student's $t$ test.

ed in these mice. We hypothesized that lack of transporters may, however, alter the homeostasis/function of Trm cells. We observed marked increases in $\mathrm{CD}^{+}, \mathrm{CD} 4^{+} \mathrm{CD} 8^{+}$, and $\mathrm{CD}^{+}$Trm cells in the PP and IELs in ABCB1a/b ABCG2 KO mice compared with WT mice, while the number of $\mathrm{T}$ cells in the spleen was comparable (Figure 6A and Supplemental Figure 6, A and B). As expected Trm cells in the IEL and liver of ABCB1a/b ABCG2 KO mice lack SP phenotype (Supplemental Figure 6, C and D). In order to better understand the differences in functional properties of these $\mathrm{T}$ cells, we compared IFN- $\gamma$ production in IEL Trm cells from these mice. Freshly isolated $\mathrm{CD} 4^{+}$and $\mathrm{CD} 8^{+}$IEL Trm cells (but not blood and splenic T cells) from transporter-deficient mice produced much more IFN- $\gamma$ than those from WT mice (Figure 6, B and C, and Supplemental Figure $6 \mathrm{E})$. Interestingly, increased IFN- $\gamma$ production in IELs of transporter-deficient mice was restricted to $\mathrm{CD} 69^{+} \mathrm{CD}_{103^{+}}$ cells and not observed in $\mathrm{CD}^{6} 9^{+} \mathrm{CD} 103^{-}$cells (Figure 6, B and $\mathrm{C}$ ). Together, these data demonstrate that $\mathrm{ABC}$ transporters may regulate homeostasis and functional properties of Trm cells in the gut.

Mobilization and adoptive transfer of Tsp cells in vivo. Adoptive transfer of $\mathrm{T}$ cells has emerged as a promising therapeutic strategy against human cancer, although there is a need to enhance infiltration and persistence of transferred cells in the tumor tissue, particularly in the setting of solid tumors. The finding that human Tsp cells have a quiescent phenotype and shared biology with Trm cells potentially makes them attractive candidates as vehicles for adoptive cellular therapy. These considerations led us to explore the effect of adoptive transfer of human Tsp cells in the context of a xeno-graft-versushost disease (xeno-GVHD) model and to explore their mobilization into circulation in patients. In order to evaluate the in vivo proliferative potential and effector function of human Tsp cells, we adoptively transferred sorted human Tsp cells and control $\mathrm{T}$ cells (total T cells) into immune-deficient NOD.Cg-Prkdc scid $I l 2 r g^{t m l W j l} / S z J$ (NSG) mice and analyzed human $\mathrm{T}$ cell engraftment and resultant tissue pathology 6 weeks following adoptive transfer. Adoptive transfer of human Tsp cells led to greater engraftment of human $\mathrm{CD} 45^{+} \mathrm{T}$ cells compared with the injection of total $\mathrm{T}$ cells (Figure 7A). This was also associated with a greater degree of tissue infiltration (particularly in the liver) and severity score for GVHD (Figure 7, B and C). 
A
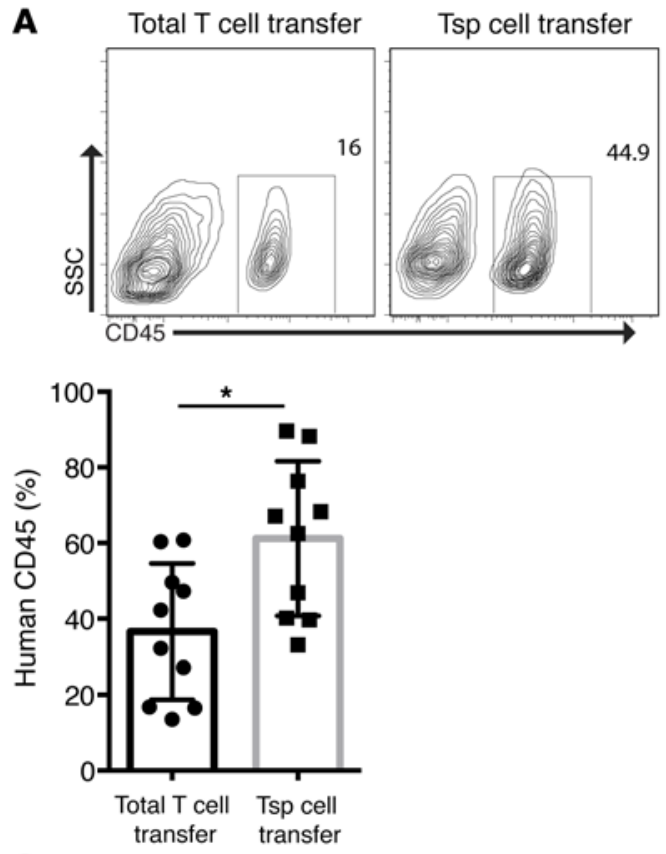

C
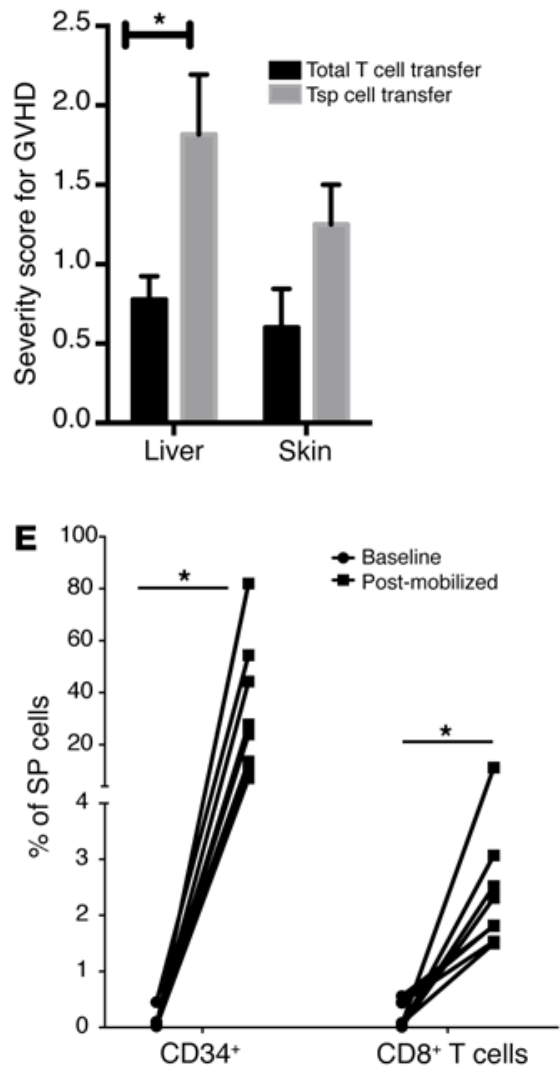

B
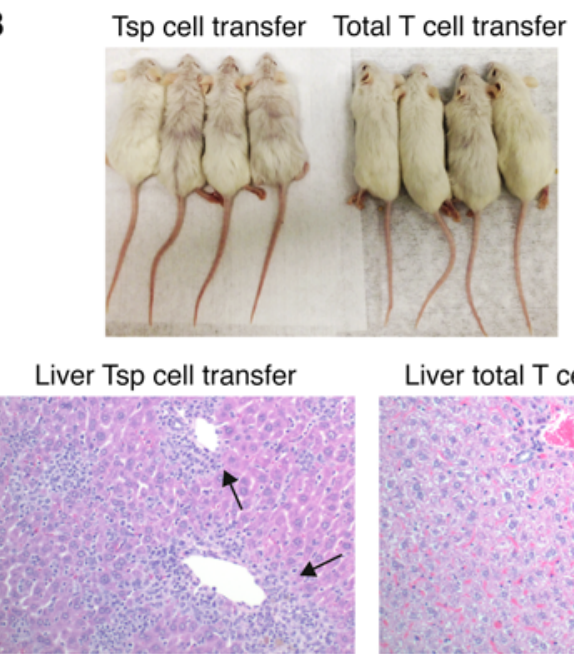

Liver total T cell transfer

Skin Tsp cell transfer

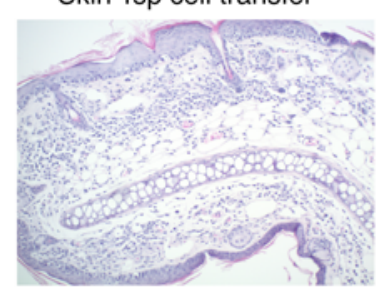

Skin total T cell transfer
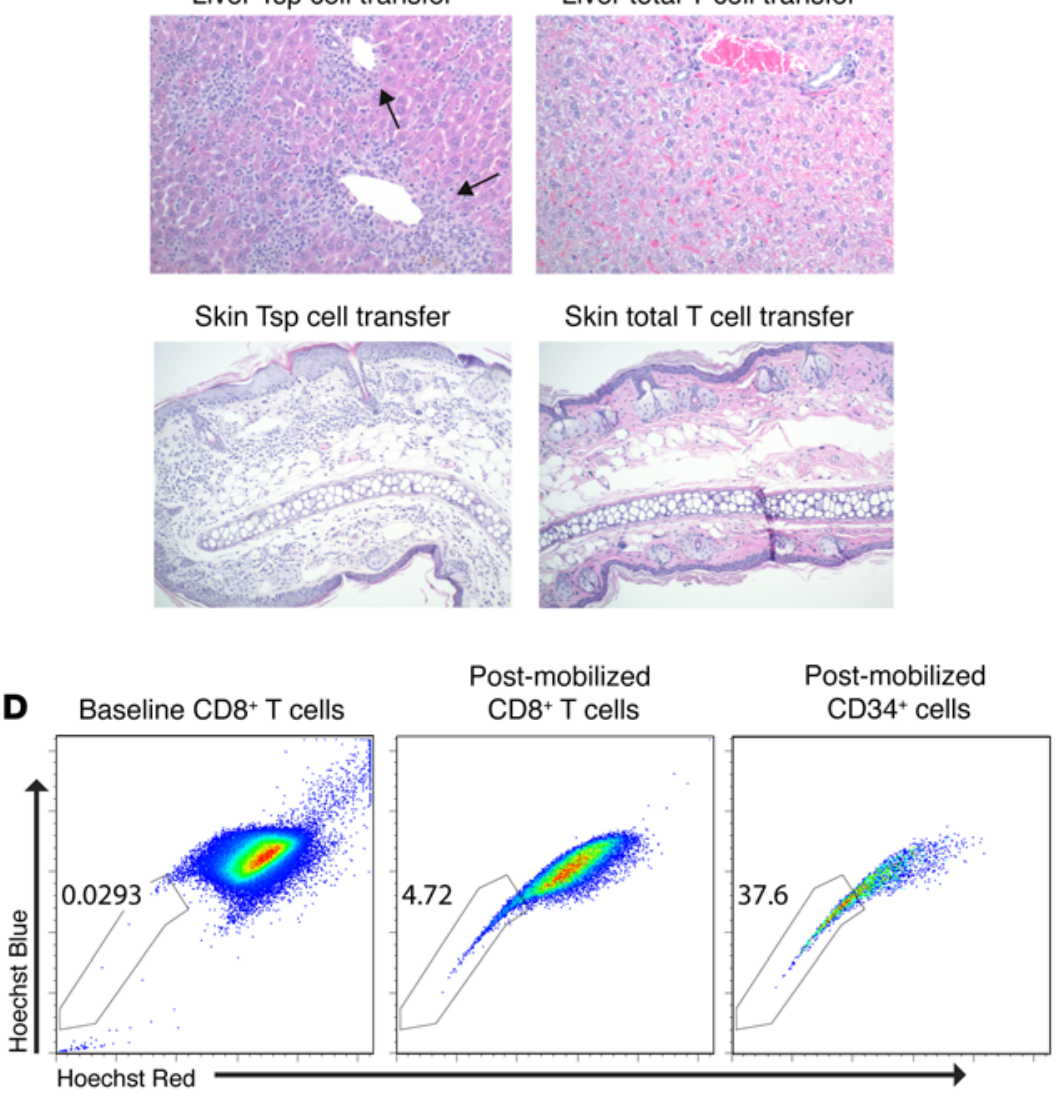

Figure 7. Human Tsp cells cause greater tissue pathology following adoptive transfer in mice and can be mobilized into circulation by plerixafor. Sorted Tsp cells and control T cells (total T cells); 100,000 of each were retroorbitally transferred into individual NSG mice and analyzed after 5 to 6 weeks. (A) FACS plot showing engraftment of human $\mathrm{CD} 45^{+} \mathrm{T}$ cells; the same is plotted in the bar graph. (B) Photographs compare skin pathology, liver, and skin histopathology in NSG mice receiving Tsp and control T cells. Original magnification $\times 200$. (C) Graph representing GVHD score for liver and skin. Experiments were repeated 3 times with 4 mice/group. (D) FACS plot represents Tsp phenotype on pre- and postmobilized CD8 $8^{+}$T cells and CD34+ ${ }^{+}$cells from human peripheral blood. (E) Graph denotes percentage of Tsp fraction in CD8 ${ }^{+}$and $C D 34^{+}$cells at baseline and after mobilization $(n=6)$. ${ }^{*} P<0.05$, by Student's $t$ test. 
The finding that human Tsp cells were capable of greater engraftment and tissue infiltration in vivo supported our hypothesis that these may be attractive vehicles to improve adoptive $\mathrm{T}$ cell therapy. However, a potential obstacle with clinical application of such a strategy is that most human Tsp cells are restricted to tissues, including the BM. Human HSCs with SP phenotype and long-term repopulation potential can be mobilized from the BM with the CXCR4 antagonist plerixafor. Therefore, we examined whether this strategy can also lead to concurrent mobilization of human Tsp cells in vivo. Injection of plerixafor led to a marked increase in circulating $\mathrm{CD}^{+} \mathrm{Tsp}$ cells within 24 hours, concurrent with mobilization of $\mathrm{CD} 4^{+} \mathrm{SP}^{+} \mathrm{HSCs}$. Together, these studies demonstrate that mobilization of human $\mathrm{CD}^{+}$Tsp cells is possible using routine methodology and, as such, can permit their potential application as a platform for adoptive $\mathrm{T}$ cell therapy in humans (Figure 7, D and E).

In order to further evaluate whether $\mathrm{CD}^{+} \mathrm{Tsp}$ cells could differentiate into Trm cells, we adoptively transferred murine Tsp cells from P14 (Thy $1.1^{+}$) chimeras into $\mathrm{Rag}^{-/-}$mice. Adoptively transferred LCMV-specific P14 (Thy1.1+) Tsp CD8 ${ }^{+}$T cells were able to generate $\mathrm{CD}^{+}$Trm cells in the gut, while the generation of Tcm cells in the spleen was very low (Supplemental Figure 7, A and B). Therefore, these data indicate that Tsp cells are able to generate Trm cells in vivo. The capacity to generate Trm cells may be particularly important for the application of adoptive $\mathrm{T}$ cell therapy as a tool to boost immunity in NLTs, such as in the setting of solid tumors.

\section{Discussion}

Here we have identified a distinct subset of human/murine slow-cycling $\mathrm{T}$ cells that efflux Hoechst dyes and are enriched within a population of $\mathrm{T}$ cells that display canonical Trm properties. T cells with a SP phenotype were enriched in tissues such as gut and skin known to be enriched in Trm cells, but were also enriched in the human BM and could even be detected in human blood. Interestingly, even circulating human Tsp cells express core genomic signatures of human Trm cells within tissues. The precise relationship between circulating Tsp cells and classic tissue-based Trm cells needs further study, although the presence of Trm cells with the vascular compartment in some tissues and traffic of T cell subsets to/from tissues is being increasingly appreciated $(5,7,30)$. Recent studies have also emphasized the presence of a quiescent tissue-resident compartment of memory $\mathrm{T}$ cells in stromal niches within the $\mathrm{BM}(31,32)$, which is consistent with these studies.

A unifying property of human/murine Tsp cells in all tissues (as well as those in human blood) was their enrichment in the $\mathrm{G}_{0}$ phase of the cell cycle, indicating a quiescent/slow-cycling phenotype. In adult stem cells, quiescence is intricately linked to long-term persistence and repopulating ability (33). Therefore, it is possible that similar biology exists within $\mathrm{T}$ cells and the presence of slow-cycling $\mathrm{T}$ cells is essential for long-term maintenance of the naive and memory $\mathrm{T}$ cell repertoire. Recent studies analyzing human Trm cells suggest that these cells may be stably maintained over decades of life with only minimal turnover and homeostatic proliferation relative to Tcm cells in lymphoid tissues, although prospective studies of human tissues are lacking to date (8). Therefore, our finding that nearly all of the Trm cells with a Tsp phenotype were in the $G_{0}$ state provides evidence that the Tsp subset may reflect the quiescent compartment in Trm cells. It is notable that this is in contrast to most memory $\mathrm{T}$ cells in lymphoid tissues, which are typically in $G_{1}$, which permits homeostatic turnover $(34,35)$. Diminished homeostatic cycling of Tsp cells was also confirmed based on the label-retaining fraction of Trm cells in histone 2B GFP mice. Label retention in these mice has also been utilized to identify quiescent compartments in other tissues (25). It is notable that gut Trm cells seem to have a greater proportion of label-retaining cells than memory $\mathrm{T}$ cells in lymphoid tissues. These studies therefore suggest that turnover in the Trm compartment may be lower than in Tcm cells. Furthermore, there is heterogeneity relating to the cell cycle even within Trm cells, and the Tsp phenotype specifically marks the label retaining subset of CD ${ }^{+}$Trm cells within tissues.

Two gene families enriched in the Tsp core gene-expression signature were $\mathrm{ABC}$ transporters and the NR4A family of transcription factors. ABC transporters are directly responsible for the SP phenotype and postulated to help protect stem cells from oxidative and genotoxic stress, although the nature of specific substrates may depend on the specific tissue (19). It is likely that these transporters may play a similar role in the context of T cells as well. This may be particularly true in the setting of Trm cells at barrier sites such as the gut, wherein they may be needed to actively efflux substrates to keep gut Trm cells in the quiescent state. Indeed, gut Trm cells from transporter-deficient mice had an inflammatory phenotype. Notably, increased IFN- $\gamma$ production by gut $\mathrm{T}$ cells in these mice was restricted to only Trm cells, but not non-Trm cells, consistent with a Trm-specific impact of transporter deficiency.

In addition to $\mathrm{ABC}$ transporters, core gene-expression signatures of Tsp cells was also enriched for the NR4A family of transcription factors. These nuclear receptors act as transcription factors to induce or repress gene expression (29). NR4A1 is activated as a part of early response, related in part to the strength of TCR signaling and implicated in regulating T cell differentiation $(28,36)$. Recently, NR4A1 was shown to regulate the expansion and effector functions of murine $\mathrm{CD}^{+}$effector $\mathrm{T}$ cells (same as Tem cells) through direct repression of IRF4 (37). Our studies suggest a role for this factor in the biology of murine Trm cells. T cells from Nur $77^{-1-}$ mice demonstrated reduced capacity to generate Trm cells, indicating that this transcription factor may be important in regulating the generation/ tissue residence of Trm cells. This dependence on NR4A1 is reminiscent of the situation in patrolling monocytes, which express all 3 NR4A genes, but depend only on NR4A1 (38).

Adoptive transfer of genetically modified T cells, either with chimeric antigen receptors (CAR) or antitumor TCRs is emerging as a promising therapeutic approach in hematologic malignancies $(39,40)$. However, most of the clinical success with CAR T cells to date is limited to patients with leukemia, and there is an unmet need to develop approaches to enhance persistence of transferred $\mathrm{T}$ cells and enhance their ability to infiltrate NLTs in order to be able to target solid tumors (39). Our findings that human/murine Tsp cells represent a quiescent $\mathrm{T}$ cell subpopulation, with a potential to be enriched in tissues over the long term in vivo and the ability to be mobilized into circulation in humans, suggest that these cells may provide an attractive platform for immunotherapy, particularly for tumors in NLTs and BM. 


\section{Methods}

Mice. C57BL/6j mice from the Jackson Laboratory and Thy-1.1+ TCR transgenic mice (41) that recognize the $\mathrm{H}-2 \mathrm{D}^{\mathrm{b}}$ gp33 epitope were used where indicated. H2B GFP mice were purchased from the Jackson Laboratory. ABCB1a/b ABCG2 KO mice and FVB-N mice were purchased from Taconic.

Mouse infections. For LCMV infections, mice were i.p. infected with $2 \times 10^{5}$ PFU LCMV-Armstrong (LCMV-Arm). For influenza infections, mice were inoculated i.n. with $0.8 \times 10^{5}$ TCID50 recombinant x31-OVA influenza virus expressing OVA $_{257-262}$ (SIINFEEKL).

Human samples. PBMCs from healthy donors were isolated from buffy coats purchased from the New York Blood Center. Gut T cells were obtained from uninvolved intestine in patients undergoing surgery for diverticular disease or gut biopsies. BM aspirates were obtained from patients with cancer undergoing diagnostic BM biopsies. In some patients, blood samples were collected before and after stem cell mobilization protocol with plerixafor.

Isolation of intestinal and skin T cells. PP were first isolated, followed by isolation of IEL and LPL. After removal of PP, intestines were cut longitudinally, and the intestinal contents along with mucus were removed by gentle scraping. The intestines were then cut into small pieces and incubated in PBS buffer containing $1 \mathrm{mM}$ EDTA, $1 \mathrm{mM}$ DTT, and 5\% FBS at $37^{\circ} \mathrm{C}$ in an incubator-shaker at $28 \mathrm{~g}$ for 15 minutes. The supernatant was collected to isolate the IELs, while the remaining intestinal pieces were incubated further in LPL/collagenase $(1 \mathrm{mg} / \mathrm{ml}) / \mathrm{DNase}$ $(1 \mathrm{U} / \mathrm{ml})$ in $\mathrm{PBS}$ solution at $37^{\circ} \mathrm{C}$ in an incubator-shaker for 10 minutes for the isolation of LPLs. Skin T cells were obtained from human skin following methods described earlier (42).

Flow cytometry. Single-cell suspensions were made from human and mouse tissues. Cell-surface FACS staining was performed in FACS buffer (PBS + 2\% FBS) by incubating cells with fluorochromeconjugated antibodies for 30 minutes on ice in the dark. After staining, the cells were washed with FACS buffer and analyzed with BD LSRII. Intracellular staining was performed using $\mathrm{BD}$ reagents following the manufacturer's protocol. Flow cytometry antibodies for human and mouse were purchased from BD, eBioscience and BioLegend. Flow cytometry data were acquired on BD LSRII with Diva software and analyzed with FlowJo 9.7.7 software (Treestar).

The following fluorescently labeled antibodies were used: anti human CD3 (clone UCHT1; BioLegend), anti human CD4 (clone RPA-T4; BD Biosciences), anti human CD8 (clone SK1; BD Biosciences), anti human CD69 (clone FN50; eBiosciences), anti human CD103 (clone B-Ly7; eBiosciences), anti human CD62L (clone DREG-56; eBiosciences), anti human CCR7 (clone G043 H7; BioLegend), anti human CD45RO (clone UCHL1; BD Biosciences), anti human IFN- $\gamma$ (clone B27; BD Biosciences), anti human CD45RA (clone HI 100; eBiosciences), anti mouse CD4 (clone RM4.5; BD Biosciences), anti mouse CD8 (clone 53-6.7; BD Biosciences), anti mouse CD69 (clone HI.2F3; eBiosciences), anti mouse CD103 (clone 2E7; eBiosciences), anti mouse CD62L (clone MEL-14; eBiosciences), anti mouse CD44 (clone IM7; eBiosciences), anti mouse Thy1.1 (clone OX-7; BD Biosciences), anti mouse CD45.2 (clone 104; eBiosciences), anti mouse CD45.1(clone A20; eBiosciences), anti mouse IFN- $\gamma$ (clone XMG1.1; BD Biosciences) and anti mouse TCR V 22 (clone B20.1; BioLegend).

Detection of Tsp cells. Hoechst staining was performed on single-cell suspension of mouse and human cells at 1 to 2 million cells $/ \mathrm{ml}$ using the method described by Goodell et al. (13). In brief, cells were incubated with Hoechst $33342(5 \mu \mathrm{g} / \mathrm{ml})$ (Sigma- Aldrich) in 2\% FCS and $1 \mathrm{mM}$ HEPES with or without verapamil $(50 \mu \mathrm{M})$ (Sigma-Aldrich) for 90 minutes at $37^{\circ} \mathrm{C}$ in an incubator in darkness with intermittent shaking. The cells were then washed in ice-cold Hoechst wash buffer and analyzed with a BD LSRII-containing UV laser.

Hoechst-Pyronin $Y$ staining. $\mathrm{G}_{0} / \mathrm{G}_{1}$ analysis was performed as previously described (24). In brief, cells were incubated in Hoechst 33342 buffer for 1 hour at $37^{\circ} \mathrm{C}$. After that, Pyronin Y $(5 \mu \mathrm{g} / \mathrm{ml}$; SigmaAldrich) was added and cells were incubated for an additional 30 minutes. Subsequently, cells were washed with ice-cold buffer and analyzed using BD-LSRII.

Adoptive transfer of human T cells into NSG mice. Human Tsp cells and control $\mathrm{T}$ cells were FACS sorted and adoptively transferred via i.v. injection with 50 to $100 \times 1,000 \mathrm{~T}$ cells/mouse. To reduce the variability in each experiment, 3 replicate mice from each group received the sorted Tsp cells or control T cells from the same donor. Mice were sacrificed at 8 to 10 weeks. Organs such as skin, liver, and small intestine were tested for tissue pathology. Spleen was harvested, and single-cell suspensions were made and stained for FACS to detect human T cell engraftment.

Adoptive transfer of murine SP CD $8^{+}$T cells. P14 (Thy $1.1^{+}$) chimeras were generated and infected with LCMV. At 30 days after infection, SP CD $8^{+} \mathrm{T}$ cells (containing both SP Thy $1.1^{+}$and polyclonal SP CD $8^{+}$ $\mathrm{T}$ cells) were sorted and adoptively transferred to RAG mice. At 3 weeks following transfer, the presence of Trm cells in the gut and $\mathrm{Tcm} /$ Tem cells in the spleen was analyzed by flow cytometry.

TCR sequencing. Genomic DNA was isolated from peripheral blood SP CD8 ${ }^{+}$T cells or MAIT cells $\left(\mathrm{CD} 8^{+} \mathrm{CD} 161^{+} \mathrm{IL} 18 \mathrm{R} \alpha^{+}\right)$, and the diversity of TCR was profiled using high-throughput sequencing of rearranged TCR $\beta$ loci from genomic DNA by Adaptive Biotechnologies as previously described (43).

Microarray analysis. RNA from sorted human SP CD $4^{+}$and $\mathrm{CD} 8^{+}$ $\mathrm{T}$ cells was amplified, labeled, and hybridized on the Affymetrix Human Genome U133 Plus 2.0 microarray chips. The data were analyzed with Gene Spring GX12.5 (Agilent Technologies) and Partek Genomics Suite (6.6) software, as previously described $(44,45)$. All original microarray data were deposited in the NCBI's Gene Expression Omnibus (GEO GSE85074).

Immunohistochemistry. Four to five mice per group (8 to 12 weeks) were studied in each experiment. Mice were adoptively transferred with Tsp and control T cells. After 4 weeks of transfer, mice were euthanized and organs (skin, liver and small intestine) were examined for GVHD. Briefly, tissue sections prepared from formalin-fixed tissues were used for H\&E staining. Severity score generated was based on tissue pathology.

Histone 2BGFP mice. H2B-GFP mice (25) were purchased from the Jackson Laboratory. Six-week-old mice were fed with Dox (Sigma-Aldrich, D9891, $2 \mathrm{mg} / \mathrm{ml}$, supplemented with sucrose at 10 $\mathrm{mg} / \mathrm{ml}$ ) added to the drinking water for 2 to 3 weeks. Mice were then infected with LCMV-Arm and kept on Dox for 5 more weeks. After 8 weeks of Dox treatment, chase was initiated by discontinuing Dox for 5 more weeks. Mice were then euthanized and analyzed for label-retaining cells.

Statistics. A 2-tailed Student's $t$ test was used to compare data between 2 groups. A P value of less than 0.05 was considered significant.

Study approval. Studies in animals were approved by the Animal Care and Use Committee, and human studies were approved by the 
Human Investigation Committee at Yale University. Human study subjects provided informed consent prior to their participation in the study.

\section{Author contributions}

CSB designed and performed research, analyzed data, and wrote the manuscript. SN performed research and analyzed data. SMG and HNN performed some experiments. RV analyzed microarray data. JAG analyzed mouse pathology. CA, JV, KMD, CCH, RAF, and DN analyzed data. SMK designed research and analyzed data. MVD designed research, analyzed data, and wrote the manuscript.

\section{Acknowledgments}

The authors thank members of the Dhodapkar lab for helpful discussions and Lin Zhang and Connor Foster for help with specimen processing. MVD is supported in part by funds from the NIH (CA106802, CA197603).

Address correspondence to: Madhav V. Dhodapkar, Yale University School of Medicine, 333 Cedar Street, New Haven, Connecticut 06515, USA. Phone: 203.785.4144; E-mail: madhav.dhodapkar@ yale.edu.
1. Hammarlund E, et al. Duration of antiviral immunity after smallpox vaccination. Nat Med. 2003;9(9):1131-1137.

2. Sallusto F, Lenig D, Förster R, Lipp M, Lanzavecchia A. Two subsets of memory T lymphocytes with distinct homing potentials and effector functions. Nature. 1999;401(6754):708-712.

3. Mueller SN, Gebhardt T, Carbone FR, Heath WR. Memory $\mathrm{T}$ cell subsets, migration patterns, and tissue residence. Annu Rev Immunol. 2013;31:137-161.

4. Gebhardt T, Wakim LM, Eidsmo L, Reading PC, Heath WR, Carbone FR. Memory T cells in nonlymphoid tissue that provide enhanced local immunity during infection with herpes simplex virus. Nat Immunol. 2009;10(5):524-530.

5. Schenkel JM, Masopust D. Tissue-resident memory T cells. Immunity. 2014;41(6):886-897.

6. Masopust D, Vezys V, Marzo AL, Lefrançois L. Preferential localization of effector memory cells in nonlymphoid tissue. Science. 2001;291(5512):2413-2417.

7. Steinert EM, et al. Quantifying Memory CD8 T Cells Reveals Regionalization of Immunosurveillance. Cell. 2015;161(4):737-749.

8. Thome JJ, et al. Spatial map of human T cell compartmentalization and maintenance over decades of life. Cell. 2014;159(4):814-828.

9. Gaide $\mathrm{O}$, et al. Common clonal origin of central and resident memory $\mathrm{T}$ cells following skin immunization. Nat Med. 2015;21(6):647-653.

10. Mackay LK, et al. The developmental pathway for CD103(+)CD8+ tissue-resident memory T cells of skin. Nat Immunol. 2013;14(12):1294-1301.

11. Fearon DT, Manders P, Wagner SD. Arrested differentiation, the self-renewing memory lymphocyte, and vaccination. Science. 2001;293(5528):248-250.

12. Luckey CJ, Bhattacharya D, Goldrath AW, Weissman IL, Benoist C, Mathis D. Memory T and memory B cells share a transcriptional program of selfrenewal with long-term hematopoietic stem cells. Proc Natl Acad Sci US A. 2006;103(9):3304-3309.

13. Goodell MA, Brose K, Paradis G, Conner AS, Mulligan RC. Isolation and functional properties of murine hematopoietic stem cells that are replicating in vivo. J Exp Med. 1996;183(4):1797-1806.

14. Weksberg DC, Chambers SM, Boles NC, Goodell MA. CD150-side population cells represent a functionally distinct population of long-term hematopoietic stem cells. Blood. 2008;111(4):2444-2451.

15. Zhou S, et al. The ABC transporter Bcrp1/ABCG2 is expressed in a wide variety of stem cells and is a molecular determinant of the side-population phenotype. Nat Med. 2001;7(9):1028-1034.

16. Doyle MJ, et al. Abcg2 labels multiple cell types in skeletal muscle and participates in muscle regeneration. JCell Biol. 2011;195(1):147-163.

17. Scharenberg CW, Harkey MA, Torok-Storb B. The ABCG2 transporter is an efficient Hoechst 33342 efflux pump and is preferentially expressed by immature human hematopoietic progenitors. Blood. 2002;99(2):507-512.

18. Fatima S, Zhou S, Sorrentino BP. Abcg2 expression marks tissue-specific stem cells in multiple organs in a mouse progeny tracking model. Stem Cells. 2012;30(2):210-221.

19. Tarling EJ, de Aguiar Vallim TQ, Edwards PA. Role of ABC transporters in lipid transport and human disease. Trends Endocrinol Metab. 2013;24(7):342-350.

20. Turtle CJ, Swanson HM, Fujii N, Estey EH, Riddell SR. A distinct subset of self-renewing human memory CD8+ T cells survives cytotoxic chemotherapy. Immunity. 2009;31(5):834-844.

21. Dusseaux M, et al. Human MAIT cells are xenobiotic-resistant, tissue-targeted, CD161hi IL-17secreting T cells. Blood. 2011;117(4):1250-1259.

22. Land RH, et al. The orphan nuclear receptor NR4A1 specifies a distinct subpopulation of quiescent myeloid-biased long-term HSCs. Stem Cells. 2015;33(1):278-288.

23. Sirin O, Lukov GL, Mao R, Conneely OM, Goodell MA. The orphan nuclear receptor Nurr1 restricts the proliferation of haematopoietic stem cells. Nat Cell Biol. 2010;12(12):1213-1219.

24. Liu Y, et al. p53 regulates hematopoietic stem cell quiescence. Cell Stem Cell. 2009;4(1):37-48.

25 . Foudi A, et al. Analysis of histone 2B-GFP retention reveals slowly cycling hematopoietic stem cells. Nat Biotechnol. 2009;27(1):84-90.

26. Wilson A, et al. Hematopoietic stem cells reversibly switch from dormancy to selfrenewal during homeostasis and repair. Cell. 2008;135(6):1118-1129.

27. Li J, Olshansky M, Carbone FR, Ma JZ. Transcriptional Analysis of T Cells Resident in Human Skin. PLoS ONE. 2016;11(1):e0148351.

28. Sekiya T, et al. Nr4a receptors are essential for thymic regulatory $\mathrm{T}$ cell development and immune homeostasis. Nat Immunol. 2013;14(3):230-237.

29. Winoto A, Littman DR. Nuclear hormone receptors in T lymphocytes. Cell. 2002;109 Suppl:S57-S66.

30. Morton AM, Sefik E, Upadhyay R, Weissleder R, Benoist C, Mathis D. Endoscopic photoconversion reveals unexpectedly broad leukocyte trafficking to and from the gut. Proc Natl Acad Sci U S A. 2014;111(18):6696-6701.

31. Alp ÖS, Radbruch A. The lifestyle of memory
CD8(+) T cells. Nat Rev Immunol. 2016;16(4):271.

32. Sercan Alp Ö, et al. Memory CD8(+) T cells colocalize with IL-7(+) stromal cells in bone marrow and rest in terms of proliferation and transcription. Eur J Immunol. 2015;45(4):975-987.

33. Nakamura-Ishizu A, Takizawa H, Suda T. The analysis, roles and regulation of quiescence in hematopoietic stem cells. Development. 2014;141(24):4656-4666.

34. Allam A, et al. The CD8+ memory T-cell state of readiness is actively maintained and reversible. Blood. 2009;114(10):2121-2130.

35. Veiga-Fernandes $\mathrm{H}$, Rocha B. High expression of active CDK6 in the cytoplasm of CD8 memory cells favors rapid division. Nat Immunol. 2004;5(1):31-37.

36. Liu ZG, Smith SW, McLaughlin KA, Schwartz LM, Osborne BA. Apoptotic signals delivered through the T-cell receptor of a T-cell hybrid require the immediate-early gene nur77. Nature. 1994;367(6460):281-284

37. Nowyhed HN, Huynh TR, Thomas GD, Blatchley A, Hedrick CC. Cutting Edge: The Orphan Nuclear Receptor Nr4a1 Regulates CD8+ T Cell Expansion and Effector Function through Direct Repression of Irf4. J Immunol. 2015;195(8):3515-3519.

38. Hanna RN, et al. The transcription factor NR4A1 (Nur77) controls bone marrow differentiation and the survival of Ly6C-monocytes. Nat Immunol. 2011;12(8):778-785.

39. June CH, Riddell SR, Schumacher TN. Adoptive cellular therapy: a race to the finish line. $\mathrm{Sci}$ Transl Med. 2015;7(280):280ps7.

40. Maus MV, Grupp SA, Porter DL, June CH. Antibody-modified T cells: CARs take the front seat for hematologic malignancies. Blood. 2014;123(17):2625-2635.

41. Pircher H, Bürki K, Lang R, Hengartner H, Zinkernagel RM. Tolerance induction in double specific T-cell receptor transgenic mice varies with antigen. Nature. 1989;342(6249):559-561.

42. Watanabe R, et al. Human skin is protected by four functionally and phenotypically discrete populations of resident and recirculating memory T cells. Sci Transl Med. 2015;7(279):279ra39.

43. Nair S, et al. Type II NKT-TFH cells against Gaucher lipids regulate B-cell immunity and inflammation. Blood. 2015;125(8):1256-1271.

44. Sehgal K, et al. Clinical and pharmacodynamic analysis of pomalidomide dosing strategies in myeloma: impact of immune activation and cereblon targets. Blood. 2015;125(26):4042-4051.

45. Das R, et al. Combination therapy with anti-CTLA-4 and anti-PD-1 leads to distinct immunologic changes in vivo. JImmunol. 2015;194(3):950-959. 